\title{
Accuracy and Complications of Computer-Designed Selective Laser Sintering Surgical Guides for Flapless Dental Implant Placement and Immediate Definitive Prosthesis Installation
}

Giovanni A. Di Giacomo, ${ }^{* \dagger}$ Jorge V. da Silva,${ }^{\dagger}$ Airton M. da Silva, ${ }^{\dagger}$ Gustavo H. Paschoal, ${ }^{\dagger}$ Patricia R. Cury, ${ }^{\ddagger}$ and Gilberto Szarf*

Background: Computer-aided dental implant placement seems to be useful for placing implants by using a flapless approach. However, evidence supporting such applications is scarce. The aim of this study is to evaluate the accuracy of and complications that arise from the use of selective laser sintering surgical guides for flapless dental implant placement and immediate definitive prosthesis installation.

Methods: Sixty implants and 12 prostheses were installed in 12 patients (four males and eight females; age range: 41 to 71 years). Lateral (coronal and apical) and angular deviations between virtually planned and placed implants were measured. The patients were followed up for 30 months, and surgical and prosthetic complications were documented.

Results: The mean \pm SD angular, coronal, and apical deviations were $6.53^{\circ} \pm 4.31^{\circ}, 1.35 \pm 0.65 \mathrm{~mm}$, and $1.79 \pm 1.01 \mathrm{~mm}$, respectively. Coronal and apical deviations of $<2 \mathrm{~mm}$ were observed in $82.67 \%$ and $58.33 \%$ of the implants, respectively. The total complication rate was $34.41 \%$; this rate pertained to complications such as pulling of the soft tissue from the lingual surface during drilling, insertion of an implant that was wider than planned, implant instability, prolonged pain, midline deviation of the prosthesis, and prosthesis fracture. The cumulative survival rates for implants and prostheses were $98.33 \%$ and $91.66 \%$, respectively.

Conclusions: The mean lateral deviation was $<1.8 \mathrm{~mm}$, and the mean angular deviation was $6.53^{\circ}$. However, $41.67 \%$ of the implants had apical deviation $>2 \mathrm{~mm}$. The complication rate was $34.4 \%$. Hence, computer-aided dental implant surgery still requires improvement and should be considered as in the developmental stage. J Periodontol 2012;83:410-419.

\section{KEY WORDS}

Dental implants; surgery, computer-assisted.

\footnotetext{
* Department of Diagnostic Imaging, School of Medicine, Federal University of São Paulo, São Paulo, Brazil.

$\dagger$ Three-Dimensional Technology Division, Renato Archer Information Technology Center Campinas, Brazil.

‡ Department of Periodontics, School of Dentistry of the Federal University of Bahia, Salvador, Brazil.
}

I horough presurgical planning is a prerequisite for successful dental implant rehabilitation ${ }^{1}$ and involves anatomic as well as prosthetic considerations. Computerized tomography (CT) scans, threedimensional (3D) surgical planning software, and rapid prototyping of surgical guides have enabled virtual planning in the field of surgery and have been integrated for improving presurgical planning. ${ }^{2}$ Computeraided dental implant surgery seems to be especially useful in cases in which implants are placed using a flapless approach. Moreover, the integration of restorative determinants into surgical planning allows for the production of the prostheses before surgery, simplifying immediate loading protocols. ${ }^{3,4}$ However, there is no conclusive evidence supporting such advantages, and many issues are open to debate.

Rapid prototyping techniques allow the production of physical models on the basis of virtual computational models. Two leading rapid prototyping technologies that are currently

doi: $10.1902 / j o p .2011 .110115$ 
in use are stereolithography (SLA) and selective laser sintering (SLS). SLA uses an ultraviolet laser to successfully "laser cure" cross-sections of a liquid resin. SLS uses a carbon dioxide laser to fuse together layers of a fine polyamide powder. Compared to SLA, SLS has the advantage of not requiring support structures because the unsintered powder provides support during the build of models. ${ }^{5}$ SLS models are opaque, whereas SLA models are translucent. ${ }^{5}$ The SLS process seems to be accurate and adequate for medical application. ${ }^{6}$

Data on the accuracy ${ }^{7,8}$ of SLA surgical guides for flapless dental implant placement and immediate prosthesis installation and the complications s,4,7-11 $^{\text {of their }}$ use for this purpose are scarce, and previously published studies on these complications involved a short follow-up (Table 1). The accuracy of SLS surgical guides for implant placement and the complications associated with their use for this purpose are not known. Therefore, the aim of the present prospective clinical study is to evaluate the accuracy of, and the complications (surgical and prosthetic) associated with, the use of SLS surgical guides for flapless dental implant placement and immediate definitive prosthesis installation.

\section{MATERIALS AND METHODS}

The study protocol was approved by the institutional ethics committee of the University Hospital of the Federal University of São Paulo, São Paulo, Brazil. Written informed consent was obtained from all of the patients.

The study was performed from January 2006 to December 2009. Twelve patients (four males and eight females; aged 41 to 71 years; mean age: 60.3 years) were recruited with sufficient bone for implant installation and indications for implant rehabilitation. The exclusion criteria were: 1 ) radiotherapy; 2) chemotherapy; 3 ) chronic systemic diseases; 4) poor oral hygiene; 5) alcohol, tobacco, or drug abuse; and 6) bruxism. Preoperative procedures were performed 4 months before the surgeries.

A single clinician (GADG), an expert in implant dentistry and computer-aided oral implant surgery, performed the virtual planning, surgeries, and prostheses installation. The protocol involved the following steps: 1) Fabrication of a radiopaque radiographic template composed of high-density barium (10\%) and varnish $(90 \%)$ that was an exact replica of the prosthesis used by the patient. The template covered the occlusal surfaces of the complete dental arch up to the coronal third of the dentition and reached the mucosa in the edentulous area. An interocclusal support (occlusal index) was prepared to separate the mandibular and maxillary arches and stabilize the template during tomography (Fig. 1A). 2) A CT scan was taken of the patient's dental arch. The radiographic template was positioned in the mouth of the patient, and a single scan was obtained using cone-beam computer tomography (CBCT). ${ }^{\S}$ The
CBCT scan was taken without interarch contact, using the occlusal index. 3) For virtual planning of the surgeries, the resulting CT image was converted into a digital imaging and communicating in medicine image, and 1.0-mm-thick sections were obtained using a computer-aided design (CAD) software. ${ }^{\|}$The digital image was imported into the planning software." Image segmentation (removal of soft tissue) was then performed, and the virtual implants were placed in the most optimal position according to the anatomy and prosthetic design (Fig. 1B). Sixty-two implants were planned. 4) For surgical guide planning and fabrication, the virtual surgical guide was created using CAD software" (Fig. 1C). SLS rapid prototyping equipment** was used to fabricate the surgical guide (Fig. 1D). 5) For implant installation, the surgeries were performed under local anesthesia and appropriate aseptic and sterile conditions. The surgical guide was positioned on the mouth, using the interocclusal index to confirm proper seating. The surgical guide was properly fixed later using two equally distributed anchor pins ${ }^{\dagger \dagger}$ (Fig. 1E). Removable titanium guide tubes were adapted to the surgical guide, and drilling was performed using sequential drills with increasing diameters (Fig. 1F). The soft tissue was not removed to expose alveolar bone for drilling. All the implants were placed using a flapless surgical technique. The surgical guide was then removed, and 62 self-taping external hex implants $^{\ddagger}$ with diameters of 3.75 or $4.0 \mathrm{~mm}$ and lengths between 10 and $15 \mathrm{~mm}$ were placed. Conical abutments $\S \S$ were screwed onto the implants with 20 $\mathrm{N} / \mathrm{cm}$ torque (Fig. 1G). 6) For prosthesis immediately after implantation, impression copings were mounted, and an impression was made using a silicone material III and an individual tray. The tray was a replica of the prosthesis used (Fig. 1H). The metal framework was laser jointed (Fig. 1I). Within 8 hours, a definitive prosthesis was delivered to the patient (Fig. 1J). Occlusion and articulation were corrected whenever necessary. 7) For postoperative follow-up, each patient received a prescription for amoxillin (500 mg; three times daily for 7 days, starting 1 hour before the surgery), ibuprofen (400 mg, three times daily for 1 day, to be taken in case of pain), and chlorhexidine rinse $(0.12 \%$, two times daily). A visit was scheduled for 24 hours postsurgery to check the bridge screws and to hand torque the screws and adjust occlusion and articulation, if required. A follow-up visit was planned at 2 weeks, and 3 , $6,12,18,24$, and 30 months post-surgery. At each

\footnotetext{
NewTom 3G, Quantitative Radiology, Verona, Italy. NTT Software, Quantitative Radiology.

ImplantViewer 1.9, Anne Solutions, São Paulo, Brazil.

Rhino 4.0, McNeel, Seattle, WA.

* Sinterstation HiQ, 3D Systems, Rock Hill, SC.

$\dagger \dagger$ Anchor pins, Nobel Biocare, Gothenburg, Sweden.

㧊 E-Fix, AS Technology, São Paulo, Brazil.

$\S \S$ Pilar Microunit, AS Technology.

\|l| Xantopren VL Plus, Heraeus Kulzer, São Paulo, Brazil.
} 


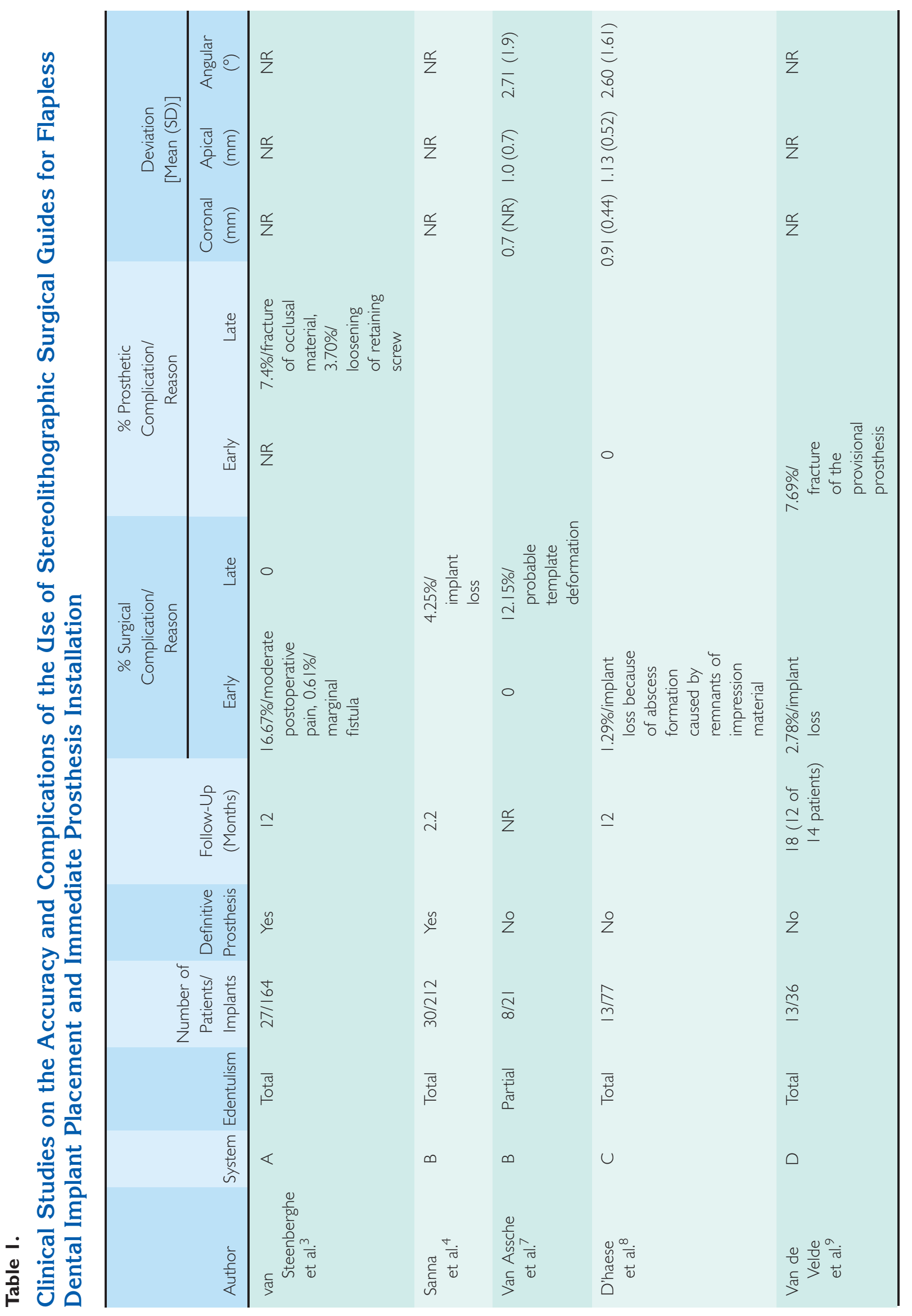




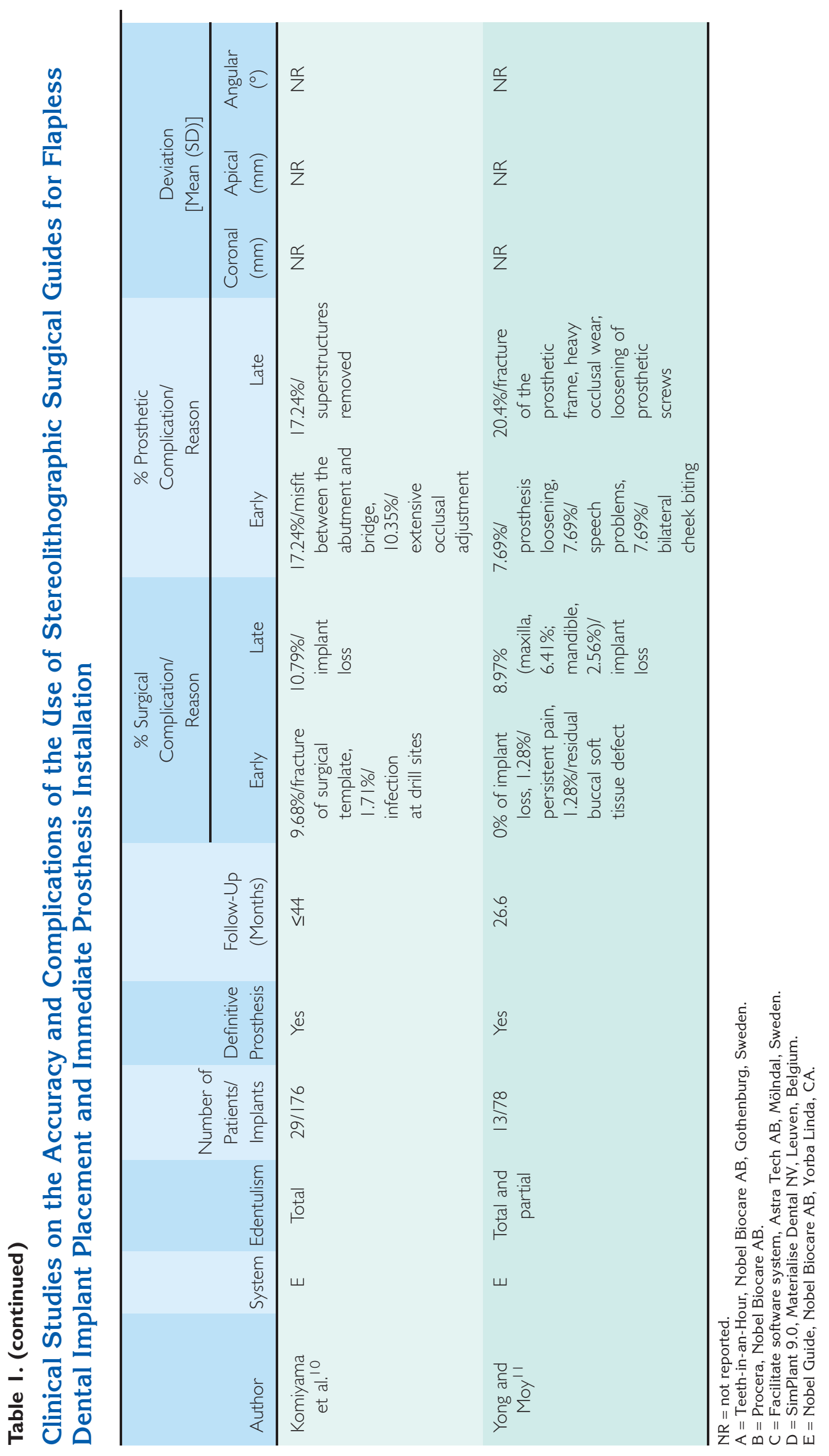



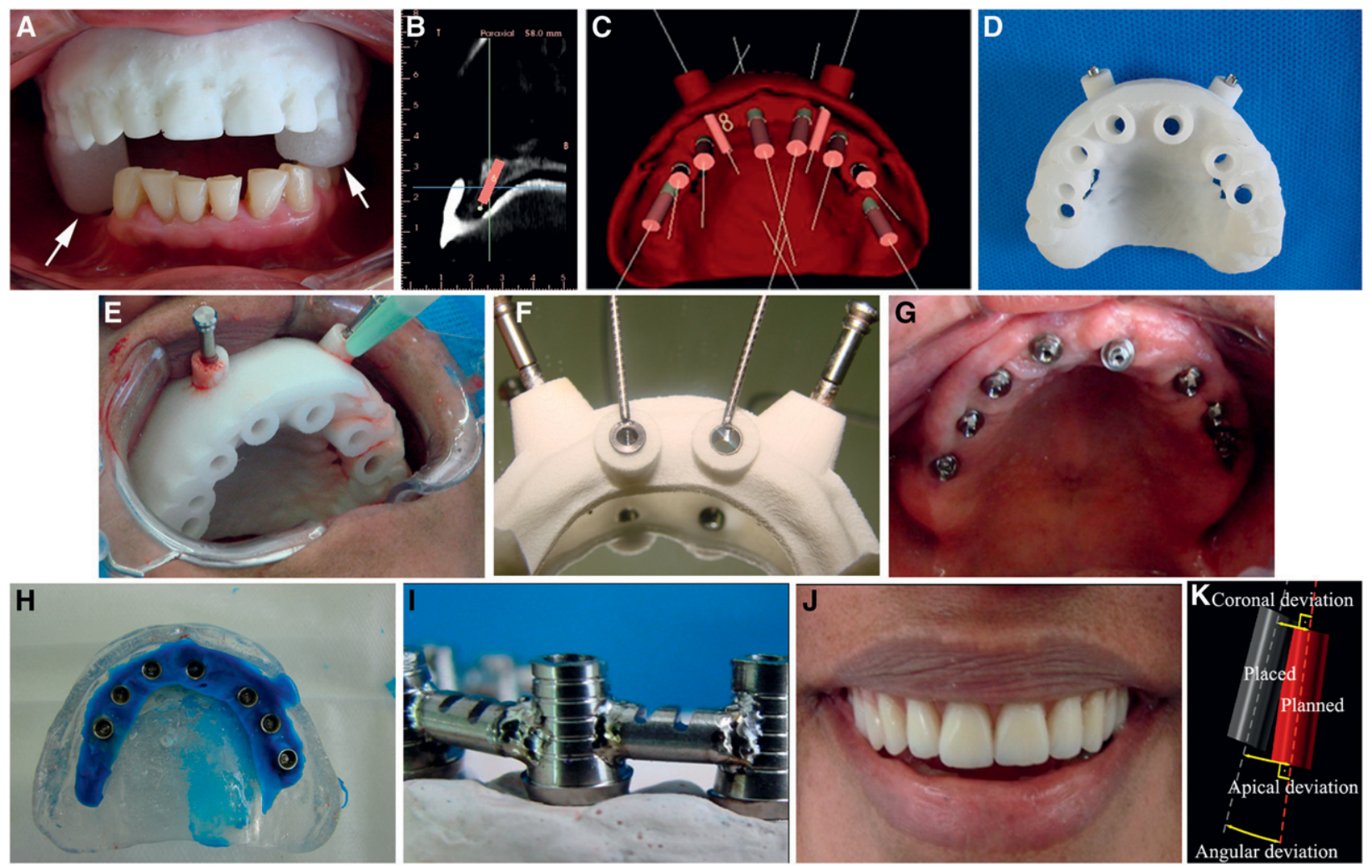

Figure I.

Computer-designed surgical guide for flapless implant placement and immediate definitive prosthesis installation. A) Radiopaque radiographic template; the arrows indicate the occlusal indices. B) Virtual implant planning. C) Virtual surgical guide showing the planned implants. D) Surgical guide fabricated using SLS. E) Surgical guide fixed using two equally distributed anchor pins. F) Details of the removable titanium guide tubes adapted in the surgical guide. G) Implants installed using a flapless approach. $\mathbf{H ) ~ I m p r e s s i o n ~ m a d e ~ u s i n g ~ a ~ s i l i c o n e ~ m a t e r i a l ~ w i t h ~ a n ~ i n d i v i d u a l ~ t r a y . ~ I ) ~ L a s e r - j o i n t e d ~ m e t a l ~}$ framework. J) Definitive prosthesis that was delivered to the patient 8 hours after implant installation. $\mathbf{K})$ 3D evaluation of the virtually planned and in vivo-placed implants.

follow-up visit, oral hygiene was evaluated and reinforced, and the screws, torque, and occlusion were checked. At the 24-month follow-up, a panoramic radiograph was taken. 8) For the second CBCT scan, within 15 days post-surgery, a new CBCT scan was taken. The CAD software Tी? was used to fuse the images of the virtually planned and actually placed implants.

\section{Accuracy Evaluation}

The virtually planned and actual implant positions were compared on the fused images by using the CAD software. Three deviation parameters were measured, as shown in Figure $1 \mathrm{~K}$. The angular deviation was measured as the 3D angle between the longitudinal axes of the planned and placed implants.

To determine the lateral deviation, we defined a reference plane that was perpendicular to the longitudinal axis of the planned implant and intersected the coronal (or apical) implant centers. The lateral deviation was calculated as the distance between the coronal (or apical) center of the planned implant and the intersection point of the longitudinal axis of the placed implant and the reference plane.

\section{Evaluation of Complications}

Patients were followed up at 24 hours, 2 weeks, 3 months, and 6 months post-surgery. Thereafter, patients were followed up every 6 months for up to 30 months. At the 6-month follow-up, the prostheses were removed. The peri-implant tissue, abutments, and implants were clinically evaluated, and $20 \mathrm{~N} / \mathrm{cm}$ torque was applied to the abutment screw. At the 24-month follow-up, a panoramic radiograph was taken and evaluated for gross bone resorption around the implants.

The following surgical complications were assessed: 1) limited access; 2) primary bone augmentation; 3 ) template fracture; 4) infection; 5) insertion of an implant that was wider/narrower or shorter than planned; 6) acute sinusitis; 7) unstable implant; 8) marginal fistula; 9) buccosinusal fistula; 10) prolonged pain; and 11) soft-tissue defects.

III Rhino 4.0, McNeel. 
The early prosthetic complications that were evaluated were misfit between suprastructure and the abutment, extensive adjustments of the occlusion, prosthesis loosening, speech problems, cheek biting, and esthetic dissatisfaction. The late prosthetic complications that were investigated were screw loosening, prosthesis fracture, occlusal wear, and pressure sensitivity. Other complications were documented on the patient charts.

\section{Statistical Analyses}

Descriptive statistics for all accuracy parameters were based on 60 placed implants. All the variables showed a normal distribution (Levene test; $P \geq 0.58$ ); therefore, a single-sample $t$ test was used to compare the obtained and planned positions. An independent-sample $t$ test was used to compare anterior and posterior implants, and mandibular and maxillary implants. Differences were considered statistically significant if the $P$ value was $\leq 0.05$.

Lateral deviation measurements were categorized into three groups: 1) $\leq 1 \mathrm{~mm}$ (clinically negligible); 2) 1 to $2 \mathrm{~mm}$ (probably clinically irrelevant); and 3) $>2$ $\mathrm{mm}$ (potentially clinically relevant). ${ }^{12}$ The complication rate was calculated for 62 planned implants and 12 prostheses. At two implant sites, the ridges were too narrow, and a flap was required to place the implants. These implants were excluded from the sample population during planning and were not included in statistical analyses. The total number of the prostheses used was 12 . The rate of cumulative survival was computed for 60 placed implants and 12 prostheses. Statistical analyses were performed using a software program."\#

\section{RESULTS}

\section{Accuracy}

Deviations between the planned and actual postoperative implant positioning were calculated for all 60 implants placed (Table 2).

The mean \pm SD angular deviation of the long axis between the planned and placed implants was $6.53^{\circ} \pm$ $4.31^{\circ}$, with a mean lateral deviation of $1.35 \pm 0.65$ $\mathrm{mm}$ at the implant neck and $1.79 \pm 1.01 \mathrm{~mm}$ at the implant apex. These deviations were statistically significant $(P<0.0000)$. Coronal and apical deviations $\leq 2 \mathrm{~mm}$ were observed in $82.67 \%$ and $58.33 \%$ of the implants, respectively (Table 3 ).

A statistically significant difference $(P=0.005)$ was found between the mean angular deviations for the maxillae and mandibles (Table 4). The difference in lateral deviation between the maxillae and mandibles was not statistically significant $(P \geq 0.17)$.

The difference between the angular deviation for posterior and anterior implants was statistically significant $(P=0.08$; Table 4$)$. However, the difference be-
Table 2.

$$
\begin{aligned}
& \text { Deviation Between Planned and Actual } \\
& \text { Implant Positions }(\mathrm{N}=60)
\end{aligned}
$$

\begin{tabular}{lccc}
\hline Deviations & Mean & SD & Range \\
\hline Angular (degrees) & 6.53 & 4.31 & 0.04 to 18.64 \\
Coronal $(\mathrm{mm})$ & 1.35 & 0.65 & 0.09 to 2.69 \\
Apical $(\mathrm{mm})$ & 1.79 & 1.01 & 0.11 to 4.00 \\
$P$ value & $<0.0000$ & $<0.0000$ & $<0.0000$ \\
\hline
\end{tabular}

Table 3.

Frequency Distribution of Coronal and Apical Deviations $(N=60)$

\begin{tabular}{lccccc}
\hline & \multicolumn{2}{c}{ Coronal Deviation } & \multicolumn{2}{c}{ Apical Deviation } \\
\cline { 2 - 6 } Deviation & Implants $(\mathrm{n})$ & $\%$ & Implants $(\mathrm{n})$ & $\%$ \\
\hline Slight $(\leq 1 \mathrm{~mm})$ & 19 & 31.00 & 15 & 25.00 \\
Moderate $(>1$ to $\leq 2 \mathrm{~mm})$ & 31 & 51.67 & 20 & 33.33 \\
Relevant $(>2 \mathrm{~mm})$ & 10 & 16.67 & 25 & 41.67 \\
\hline
\end{tabular}

tween the lateral deviations of the anterior and posterior implants was not statistically significant $(P \geq 0.51)$.

\section{Complications}

The cumulative survival rates for implants and prostheses were $98.33 \%$ and $91.66 \%$, respectively, at the 30-month follow-up. The total complication rate was $34.41 \%$, with a $17.74 \%$ surgical complication rate and a $16.67 \%$ prosthetic complication rate (Table 5 ).

Surgical complications. Three implants were removed shortly after insertion. Primary stability was not achieved for two implants placed in the tuber area. These implants were removed during the implant installation surgery and were not replaced. One patient reported severe postoperative pain. The implant was in close proximity to the nasopalatine nerve and was removed 1 week after installation.

Soft tissue from the lingual surface was pulled during drilling at four implant sites. However, postoperative dehiscence did not occur. The planned implant length was respected during the surgical procedure. However, at the time of surgery, the width of four implants differed from what was planned. Wider implants were chosen to improve the primary stability of the implants.

Complications, such as surgical template fracture or metal tube detachment, did not occur during the

\#\# Statistica 6.0, Statsoft, Tulsa, OK. 
Table 4.

\section{Position Deviation of the Implants Placed in the Maxillae and Mandibles and in Anterior and Posterior Areas $(\mathrm{N}=60)$}

\begin{tabular}{lclll}
\hline & & \multicolumn{3}{c}{ Deviation [Mean (SD)] } \\
\cline { 3 - 5 } Position of the Implants & Implants $(\mathrm{n})$ & Angular $\left(^{\circ}\right)$ & Neck $(\mathrm{mm})$ & Apex $(\mathrm{mm})$ \\
\hline Maxilla & 22 & $8.54(4.20)$ & $1.51(0.62)$ & $1.86(1.07)$ \\
Mandible & 38 & $5.37(3.98)$ & $1.26(0.66)$ & $1.75(0.99)$ \\
$P$ value & & 0.005 & 0.17 & 0.70 \\
Anterior & 51 & $6.12(4.12)$ & $1.38(0.66)$ & $1.81(1.01)$ \\
Posterior & 9 & $8.87(4.86)$ & $1.22(0.65)$ & $1.69(1.06)$ \\
$P$ value & & 0.08 & 0.51 & 0.74 \\
\hline
\end{tabular}

drilling procedure. Complications, such as infection at implant drill sites, hemorrhages, sinusitis, nerve injuries, soft-tissue defects, or bone resorption surrounding the implants, were not observed. Primary bone augmentation was not required. In cases in which there was limited access in the posterior areas, the implants were slightly tilted in the mesial direction. Two patients developed slight gingival inflammation during the follow-up, which resolved after professional prophylaxis and reinstructing the patients regarding plaque control.

Prosthetic complications. In one case, midline deviation of the prosthesis was observed. However, the patient was not dissatisfied with the esthetic appearance of the prosthesis.

A late prosthetic complication was observed in one patient 30 months after the installation: a resin fracture was observed in the vestibular area of the total prosthesis. The metal framework was not damaged. In one case, a prosthetic complication was observed wherein the prosthesis required extensive occlusal adjustment. Because resin distortion had occurred during the manufacturing process and was not related to the computer-aided implant surgery, it was not included in the complication rate. No other early or late prosthetic complications were observed.

\section{DISCUSSION}

In the present study, the mean lateral deviation is $<1.8$ $\mathrm{mm}$, and the mean angular deviation is 6.53. However, $41.67 \%$ of the implants had an apical deviation $>2 \mathrm{~mm}$. Furthermore, the total complication rate was $34.41 \%$, and the cumulative survival rates for implants and prostheses were $98.33 \%$ and $91.66 \%$, respectively.

Computer-assisted implant planning and subsequent template-guided implant placement must be highly accurate for optimal preoperative diagnostics and planning and, consequently, for developing a pre- dictable procedure for implantation and prosthetic rehabilitation. In the present study, coronal (mean $\pm S D$, $1.35 \pm 0.65 \mathrm{~mm})$, apical $(1.79 \pm 1.01 \mathrm{~mm})$, and angu$\operatorname{lar}\left(6.53^{\circ} \pm 4.31^{\circ}\right)($ Table 2$)$ deviations are higher than those reported for other clinical studies in which flapless surgery was performed. ${ }^{7,8}$ However, the accuracy obtained here was superior to that obtained in our previous study ${ }^{13}$ involving another computer-aided system and flaps for implant installation. In our previous study, the coronal, apical, and angular deviations were $1.45 \pm 1.42 \mathrm{~mm}$ (mean \pm SD), $2.99 \pm 1.77 \mathrm{~mm}$, and $7.25^{\circ} \pm 2.67^{\circ}$, respectively..$^{13}$ This increase in accuracy may be associated with the learning curve of the professionals, fastening of the surgical guides by using anchor pins, and the use of a single surgical guide.

The accuracy outcome obtained in the present study was categorized as described in a previous study. ${ }^{12}$ Therefore, a deviation $>2 \mathrm{~mm}$ was considered clinically significant because $2 \mathrm{~mm}$ is the recommended safety margin around vital structures. ${ }^{12,14}$ Considering $2 \mathrm{~mm}$ as the cutoff point for surgical relevance, $16.67 \%$ and $41.67 \%$ of the implants showed significant coronal and apical (Table 3 ) deviations, respectively, which indicates that the technique requires additional refinement. These rates were higher than those reported previously (coronal deviation, 19\%; apical deviation, 25\%). ${ }^{12}$ Although implant placement should be highly accurate for avoiding injuring essential anatomic structures, a "universally" acceptable deviation value cannot be defined. This is because, in some clinical situations, even deviations $<2 \mathrm{~mm}$ might cause injury to essential anatomic structures, whereas in other situations, implant malposition can be tolerated. However, a 2-mm deviation may result in significant prosthetic misfit when the prosthesis has been fabricated on the basis of virtually planned implants.

The differences between the accuracy obtained in our study and that reported in the literature ${ }^{7,8}$ may 
Table 5.

\section{Surgical and Prosthetic Complications for 62 Planned Implants in 12 Patients}

\begin{tabular}{|c|c|}
\hline Type & $\begin{array}{c}\text { Number of Cases } \\
\text { (\% of Total) }\end{array}$ \\
\hline \multicolumn{2}{|l|}{ Surgical } \\
\hline Limited access & 0 \\
\hline Primary bone augmentation & 0 \\
\hline $\begin{array}{l}\text { Pulling of the soft tissue from } \\
\text { the lingual surface }\end{array}$ & $4(6.45)$ \\
\hline Fracture of template & 0 \\
\hline Infection & 0 \\
\hline $\begin{array}{l}\text { Insertion of wider implant than } \\
\text { planned }\end{array}$ & $4(6.45)$ \\
\hline $\begin{array}{l}\text { Insertion of shorter implant than } \\
\text { planned }\end{array}$ & 0 \\
\hline Acute sinusitis & 0 \\
\hline Implant instability & $2(3.22)$ \\
\hline Marginal fistula & 0 \\
\hline Buccosinusal fistula & 0 \\
\hline Prolonged pain & | ( $1.6 \mid)$ \\
\hline Soft-tissue defect & 0 \\
\hline Total & II (I7.74) \\
\hline \multicolumn{2}{|l|}{ Prosthetic } \\
\hline $\begin{array}{l}\text { Misfit between suprastructure } \\
\text { and abutment }\end{array}$ & 0 \\
\hline Extensive adjustments of the occlusion & 0 \\
\hline Prosthesis loosening & 0 \\
\hline Speech problems & 0 \\
\hline Cheek biting & 0 \\
\hline $\begin{array}{l}\text { Midline deviation of the prosthetic } \\
\text { rehabilitation }\end{array}$ & I (8.33) \\
\hline Esthetic dissatisfaction & 0 \\
\hline Screw loosening & 0 \\
\hline Prosthesis fracture & I (8.33) \\
\hline Occlusal wear & 0 \\
\hline Pressure sensitivity & 0 \\
\hline \multirow[t]{2}{*}{ Total } & $2(16.67)$ \\
\hline & $13(34.41)$ \\
\hline
\end{tabular}

be attributable to many reasons: 1) Micromotion of the titanium guide tubes, which is clinically imperceptible, may have occurred inside the guide perforations. 2) The titanium guide tubes were $0.2 \mathrm{~mm}$ broader than the drills, which may have resulted in an angle deviation of $\leq 2.3^{\circ} .3$ ) Here, an earlier type of CBCT device was used, which had low-resolution diagnostic images and low segmentation accuracy. ${ }^{15}$ In contrast, Van Assche et al. ${ }^{7}$ and D'haese et al. ${ }^{8}$ used a new-generation tomographic device and dual scanning (template and template plus patient), which yields a more accurate image and therefore more accurate surgical guide fabrication. 4) Misplacement of radiographic templates during scanning and misplacement or instability of surgical guides may have resulted in deviation to some extent. 5) The implants were placed freehand into the site of guided osteotomy, whereas in the two studies ${ }^{7,8}$ mentioned previously, insertion was guided by the fixture mount. A fixture mount was not available for the present system when the study began. Accordingly, surgically guided placement of implants is more accurate than freehand placement. ${ }^{16}$ Another possible source of variation is deformation of the surgical guide during prototyping. Deformation during SLA prototyping has been reported. ${ }^{17}$ However, here we used SLS prototyping. An error of $1.79 \%$ to $2.10 \%$ for SLS models was noted. ${ }^{6,7,18}$ Because the errors were cumulative, all the steps of the protocol may have contributed to the deviation.

In the present study, a significantly higher angular deviation is detected in the maxilla than in the mandible, which is in agreement with the results reported by Ozan et al. ${ }^{19}$ Valente et al. ${ }^{12}$ reported a higher accuracy for the maxilla than for the mandible. Our results are in agreement with those of D'haese et al. ${ }^{8}$ in that even we observed a slight tendency for higher angular deviation in the posterior implants than in the anterior implants. In the present study, this observation is not related with the mesial tilting of the drill in the posterior area, which was performed to compensate for limited mouth opening; this is because this mesial inclination was included in virtual planning. It was difficult to adapt three angled abutments on the posterior areas because of limited mouth opening.

Discrepancies between the planned and actual implant positions may be associated with the two more prevalent prosthetic complications reported in the literature: 1$)$ restoration misfit $(7.2 \%)$; and 2 ) extensive occlusal adjustments $(4.3 \%){ }^{20}$ In the present study, the impression is taken after the implant is installed; this compensates for discrepancies between the planned and actual implant positions, thereby reproducing the actual implant position and completely preventing these complications. In the single case in which extensive occlusal adjustment was required, the resin distortion occurred during the manufacturing process and was not related to the computer-aided implant surgery technique. This approach may also reduce prosthesis-induced tension on the implants. We encountered other complications; the total complication rate was $34.41 \%$ (Table 5 ). The surgical complication rate $(17.74 \%)$ was higher than that reported in a recent systematic review $(9.1 \%$ of 428 patients). ${ }^{20}$ In contrast, the prosthetic complication rate $(16.7 \%)$ was less than that reported in the literature (total prosthetic complication rate, 30.8\%: early complication rate, $18.8 \%$; late complication rate, $12 \%) .{ }^{20}$ Komiyama et al. ${ }^{10}$ reported a higher rate of surgical or technical complications (42\%), and they differ from those observed in the present study (Table 1). In our study, the impression was made immediately after implantation, and the metal framework was 
joined to the prosthesis in the mouth; however, in their study, the prosthesis was fabricated completely in the laboratory on the basis of virtually planned implants. These factors may help explain the differences in the results.

According to the literature, the most frequent surgical complication is limited access, which is not observed in the present study. Only nine implants were installed in the posterior area, and when probable limited access was observed during patient examination, the planned implants as well as the placed implants were slightly tilted in the mesial direction. Low rates of implants that are wider than planned have been reported. ${ }^{20}$ Here, when 3.75 -mm implants showed poor initial stability, they were removed and $4.00-\mathrm{mm}$ implants were inserted, which explains the higher rate of wider implants than planned in our study (Table 5). Two $3.75-\mathrm{mm}$ implants placed in the tuber area did not have initial stability; these implants were removed and were not replaced. The prosthetic treatment was not compromised in either case. We think that the special care taken in achieving initial implant stability may explain the high implant survival rate reported in the present study. Pulling of the soft tissue from the lingual surface may have occurred because of incomplete covering of the mucosa by the surgical guides in such areas. This complication was not reported previously in studies using a flapless approach. ${ }^{3,4,9-13}$

The main issue with guided surgery is the seating of the surgical guide. Maladjustment between surgical guides and mucosa was not clinically perceptible. Fixation of the surgical guides by using two equally distributed anchor pins stabilized the guides, but manual press was also required. The use of additional pins might improve guide stability.

Fracture of the acrylic resin was observed in one case at the 30-month follow-up (Table 5). However, the metal framework was not damaged and was used in the new prosthesis. Here, cylindrical titanium bars with a 3-mm diameter were soldered using a laser. This process seems to provide a rigid framework, which was resistant to fracture during the 30-month period and also provided a rigid connection between the implants.

It has been suggested that the key element for successful implants may be immediate and rigid connection of implants. ${ }^{4}$ When micromotion occurs, stem cells in the osseous wound differentiate to fibroblasts and form scar tissue around the implant, thus inhibiting osseointegration. ${ }^{21}$

Despite the implant deviation and complications, the cumulative survival rates of the implants and prostheses were $98.33 \%$ and $91.66 \%$, respectively, at the 30 -month follow-up. Survival rates of $90 \%$ to $100 \%$ after a 12 to 60 month follow-up period were reported in seven clin- ical studies wherein implants were restored immediately after flapless implantation procedures.

\section{CONCLUSIONS}

Therefore, we conclude that the use of SLS surgical guides for flapless dental implant placement and immediate definitive prosthesis installation resulted in a mean lateral deviation of $<1.8 \mathrm{~mm}$ and a mean angular deviation of $6.53^{\circ}$. However, $41.67 \%$ of the implants had apical deviation $>2 \mathrm{~mm}$. The total rate of surgical and prosthetic complications was $34.41 \%$. Hence, computer-aided dental implant surgery should still be considered as being in the developmental stage. Global planning and the transfer approach still need to be improved to reduce inaccuracies and complications. Additional long-term evaluation of implant survival, bone loss, and clinical complications is required.

\section{ACKNOWLEDGMENTS}

The authors thank Daniel Takanori Kemmoku and Cesar Augusto Rocha Laureti, Fellowships in Bioengineering, Three-Dimensional Technology Division, Renato Archer Information Technology Center, Campinas, Brazil, for the technical assistance during the virtual planning of the surgeries; AS Technology, São Paulo, Brazil for the donation of the implants; and Dr. Camila Altran, private practice, São Paulo, Brazil for surgical assistance. The authors report no conflicts of interest related to this study.

\section{REFERENCES}

1. Jacobs R, Adriansens A, Naert I, Quirynen M, Hermans $\mathrm{R}$, Van Steenberghe D. Predictability of reformatted computed tomography for pre-operative planning of endosseous implants. Dentomaxillofac Radiol 1999;28: 37-41.

2. Ganz SD. Presurgical planning with CT-derived fabrication of surgical guides. J Oral Maxillofac Surg 2005; 63(Suppl. 92):59-71.

3. van Steenberghe D, Glauser R, Blombäck $U$, et al. A computed tomographic scan-derived customized surgical template and fixed prosthesis for flapless surgery and immediate loading of implants in fully edentulous maxillae: A prospective multicenter study. Clin Implant Dent Relat Res 2005;7 (Suppl. 1):S111-S120.

4. Sanna AM, Molly L, van Steenberghe D. Immediately loaded CAD-CAM manufactured fixed complete dentures using flapless implant placement procedures: A cohort study of consecutive patients. J Prosthet Dent 2007;97:331-339.

5. Berry E, Brown JM, Connell M, et al. Preliminary experience with medical applications of rapid prototyping by selective laser sintering. Med Eng Phys 1997;19:90-96.

6. Silva DN, Gerhardt de Oliveira M, Meurer E, Meurer MI, Lopes da Silva JV, Santa-Bárbara A. Dimensional error in selective laser sintering and 3D-printing of models for craniomaxillary anatomy reconstruction. J Craniomaxillofac Surg 2008;36:443-449.

7. Van Assche $N$, van Steenberghe D, Quirynen $M$, Jacobs R. Accuracy assessment of computer-assisted 
flapless implant placement in partial edentulism. $J$ Clin Periodontol 2010;37:398-403.

8. D'haese J, Van De Velde T, Elaut L, De Bruyn H. A prospective study on the accuracy of mucosally supported stereolithographic surgical guides in fully edentulous maxillae [published online ahead of print November 10, 2009]. Clin Implant Dent Relat Res. doi: 10.1111/j.1708-8208.2009.00254.X.

9. Van de Velde T, Sennerby L, De Bruyn H. The clinical and radiographic outcome of implants placed in the posterior maxilla with a guided flapless approach and immediately restored with a provisional rehabilitation: A randomized clinical trial. Clin Oral Implants Res 2010; 21:1223-1233.

10. Komiyama A, Klinge B, Hultin M. Treatment outcome of immediately loaded implants installed in edentulous jaws following computer-assisted virtual treatment planning and flapless surgery. Clin Oral Implants Res 2008;19:677-685.

11. Yong LT, Moy PK. Complications of computer-aideddesign/computer-aided-machining-guided (NobelGuide) surgical implant placement: An evaluation of early clinical results. Clin Implant Dent Relat Res 2008;10: 123-127.

12. Valente F, Schiroli G, Sbrenna A. Accuracy of computer-aided oral implant surgery: A clinical and radiographic study. Int J Oral Maxillofac Implants 2009;24: 234-242.

13. Di Giacomo GA, Cury PR, de Araujo NS, Sendyk WR, Sendyk CL. Clinical application of stereolithographic surgical guides for implant placement: Preliminary results. J Periodontol 2005;76:503-507.

14. Worthington $P$. Injury to the inferior alveolar nerve during implant placement: A formula for protection of the patient and clinician. Int J Oral Maxillofac Implants 2004;19:731-734.

15. Loubele M, Maes F, Jacobs R, van Steenberghe D, White SC, Suetens P. Comparative study of image quality for
MSCT and CBCT scanners for dentomaxillofacial radiology applications. Radiat Prot Dosimetry 2008;129: 222-226.

16. Park C, Raigrodski AJ, Rosen J, Spiekerman C, London RM. Accuracy of implant placement using precision surgical guides with varying occlusogingival heights: An in vitro study. J Prosthet Dent 2009;101: 372-381.

17. Stumpel LJ. Deformation of stereolithographically produced surgical guides: An observational case series report [published online ahead of print February 11, 2010]. Clin Implant Dent Relat Res. doi: 10.1111/ j.1708-8208.2010.00272.X.

18. Ibrahim D, Broilo TL, Heitz C, et al. Dimensional error of selective laser sintering, three-dimensional printing and PolyJet models in the reproduction of mandibular anatomy. J Craniomaxillofac Surg 2009;37: 167-173.

19. Ozan O, Turkyilmaz I, Yilmaz B. A preliminary report of patients treated with early loaded implants using computerized tomography-guided surgical stents: Flapless versus conventional flapped surgery. J Oral Rehabil 2007;34:835-840.

20. Schneider D, Marquardt P, Zwahlen M, Jung RE. A systematic review on the accuracy and the clinical outcome of computer-guided template-based implant dentistry. Clin Oral Implants Res 2009;20(Suppl. 4):73-86.

21. Szmukler-Moncler S, Salama H, Reingewirtz Y, Dubruille $\mathrm{JH}$. Timing of loading and effect of micromotion on bone-dental implant interface: Review of experimental literature. J Biomed Mater Res 1998;43:192-203.

Correspondence: Giovanni Di Giacomo, Av. Brigadeiro Luis Antonio, 2504 conj. 102, São Paulo, SP, CEP $01402-$ 000, Brazil. E-mail: giovannidi@uol.com.br.

Submitted February 24, 2011; accepted for publication July 18, 2011. 